\title{
High speed imaging of a supersonic waterjet flow
}

by I.A. Znamenskaya, Y.N. Shirshov, E.Y. Koroteeva, A.M. Novinskaya, N.N. Sysoev.

\author{
Lomonosov Moscow State University, 119991, Moscow, Russia, znamen@phys.msu.ru
}

\begin{abstract}
This work studies the jet formation process and the flow developing from the waterjet cutting head. In experiments, the high speed imaging of the supersonic jet (camera Photron FASTCAM SA5 with a frame rate of 100.000 $\mathrm{fps}$ ) is complemented with the thermographic measurements conducted using an infrared camera FLIR Systems SC7700 with a frame rate of up to $400 \mathrm{fps}$. This study aims to provide new knowledge about two-phase flows under extreme conditions, and is of particular importance for waterjet design optimization.
\end{abstract}

\section{Introduction}

Abrasive water jet (AWJ) material treatment technology is used in a huge number of industrial technological processes. Hard materials like metal, glass, ceramic, polyethylene and others can be processed with abrasive waterjet technics. High-speed water and abrasive particles mixture act as a working body in this technology. A number of experimental researches in the past years were aimed to study abrasive waterjet processing as well as flows in different parts of the machine: jet-forming orifice, which creates a high-pressure jet, a focusing accelerating tube where abrasive particles receive jet impulse. These works give new information about multiphase flows under extreme conditions and device engineering design optimization [1, 2]. At the same time, jet initiation process, being important in the analysis of material erosion, is not well studied. Starting process at supersonic jet leader speed may be accompanied by shock wave formation and processed surface deformation; it can be visualized by the shadow method of flow visualization [3].

Heating of the processed material and the jet itself is an important problem since it affects the cutting accuracy and the material deformation. The feasibility of monitoring of the AWJ cutting process by IR thermography was first studied by Kovacevic et al. [4]. It was reported that IR thermography is suitable for visualization of cutting mechanisms in opaque materials, but they concluded that the change in traverse speed yields only negligible effect on the temperature distribution in the workpiece. Monitoring method of abrasive water jet cutting process using the infrared thermography was used to observe the cutting front in the workpiece and to determine the working efficiency of the material removal process [5].

In the present paper, the jet initiation process was recorded using a high-speed camera and a shadow method; simultaneously, the thermographic measurements were conducted using an infrared camera. The aim of the research was to study the launching dynamics and thermal regime of the waterjet cutting machine jet. The visualized IR radiation map may be used to gain two different types of information regarding the impacting water flow or the surface impacted by the jet.
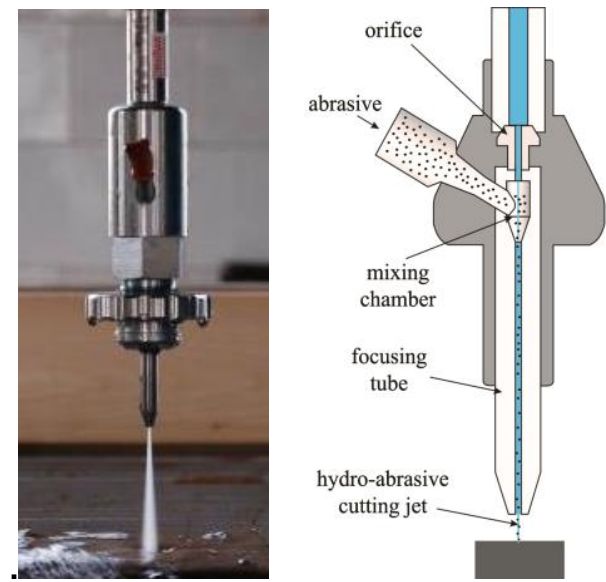

Fig. 1. Photograph of the waterjet cutting process (left) and schematic of the waterjet cutting head (right).

\section{Experimental setup}

In this work, an industrial waterjet cutting machine (model Flow WaterJet Mach3, Fig. 1) is tested. A high-speed water jet flow without abrasive is generated under the pressure of $400 \mathrm{MPa}$ and is expelled from the focusing tube. The 


\subsection{1/qirt.2016.158}

waterjet cutting head and Industrial waterjet cutting machine model Flow WaterJet Mach 3 is used to generate a highspeed water jet. Machine is able to produce a high-speed water flow, which comes through the 0.35 mm diameter orifice under the $100 \mathrm{MPa}$ or $400 \mathrm{MPa}$ pressure and has a speed about three times more than the sound speed in air [4,5]. This flow passes an abrasive mixing chamber and a $74 \mathrm{~mm}$ length focusing tube with a $1.05 \mathrm{~mm}$ channel and spreads into air under normal conditions. The diameter of a jet center zone is about $1 \mathrm{~mm}$ [6].

Here, the jet outflow process from the focusing tube under the pressure of $400 \mathrm{MPa}$ with and without abrasive is studied.

Fig. 1 shows the waterjet cutting head. The highly pressed liquid is ejected from a pump; it travels through an abrasive mixing chamber and a focusing tube, then outflows to the air as a mixture of water and abrasive particles (or only water), which is a working body in the abrasive waterjet technology of material cutting. Finally, it impacts a bump stop.

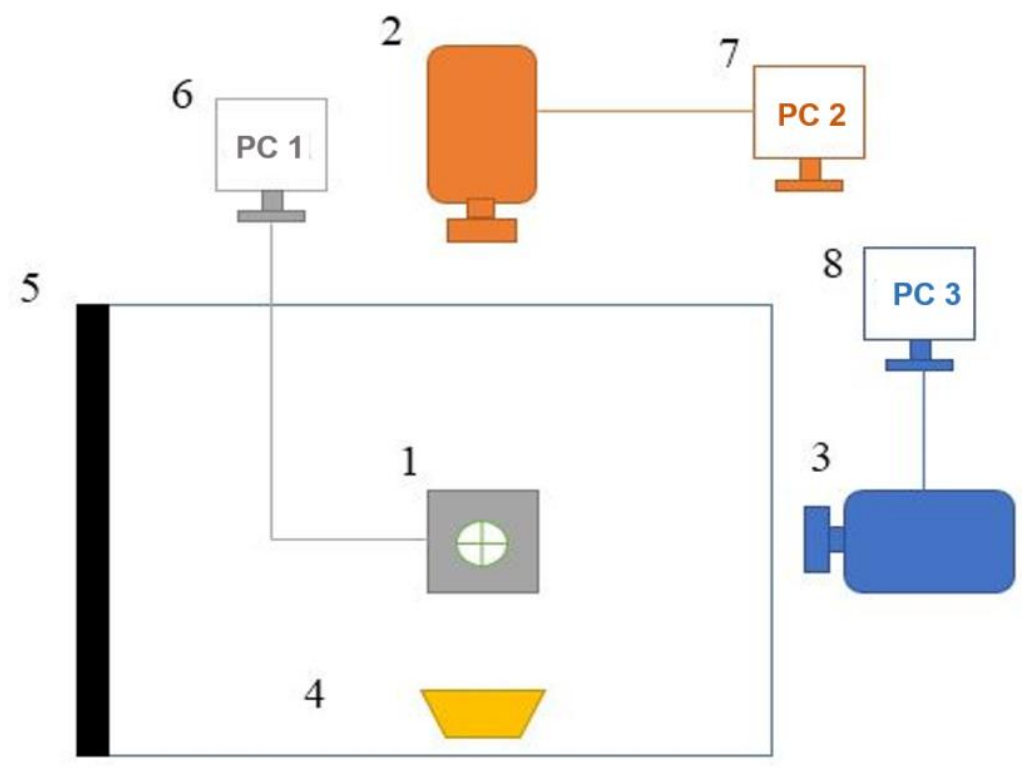

Fig. 1. Experiment scheme. 1 - Flow WaterJet Mach3 machine, 2 - high speed camera Photron FASTCAM SA5, 3 FLIR SC7700 camera, 4 - probe lamp, 5-black screen; 6-PC for cutting machine control, 7, 8 - PCs for cameras control.

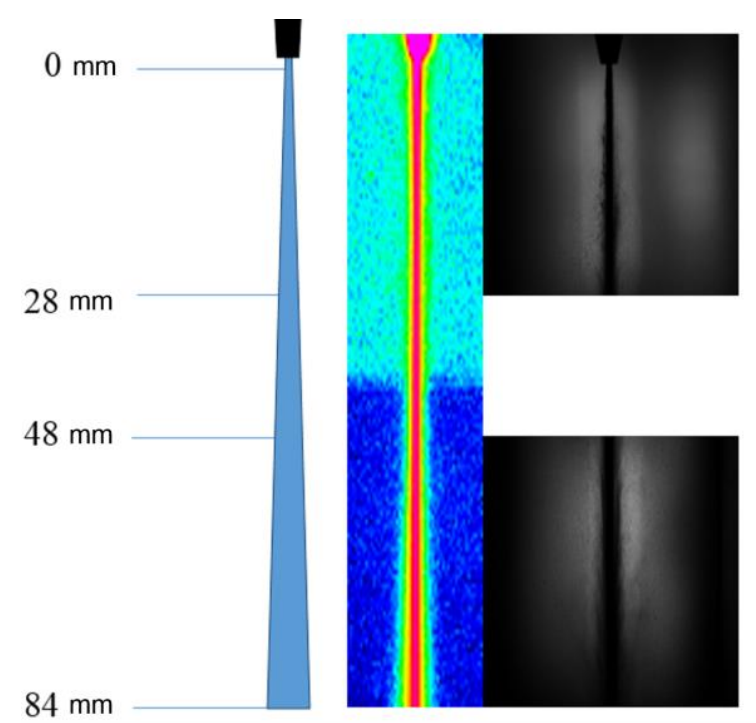

Fig. 3. Location of the jet area recorded by high-speed camera and a thermal imager. 


\subsection{1/qirt.2016.158}

Experimental scheme is shown in Fig. 2. Two visualization methods were used to study the high-speed jet flow: shadowgraphy and thermography. A high-speed optical camera Photron FASTCAM SA5, capable of recording videos up to 2 seconds with 1 million fps, was used with a Nikon AF NIKKOR lens to capture the jet forming process. Direct measurement of the water jet temperature was not carried out due to extreme conditions; instead, the thermographic videos were.recorded. The studies were conducted using a FLIR SC7700 thermal imager system, having a spectral measurement range of 3.7-4.8 $\mu \mathrm{m}$ (at $60 \%$ of a maximum sensitivity level), and a frame rate up to $115 \mathrm{~Hz}$ in a full-frame mode. Fig. 3 shows the location of the shooting jets of high-speed camera and a thermal imager.
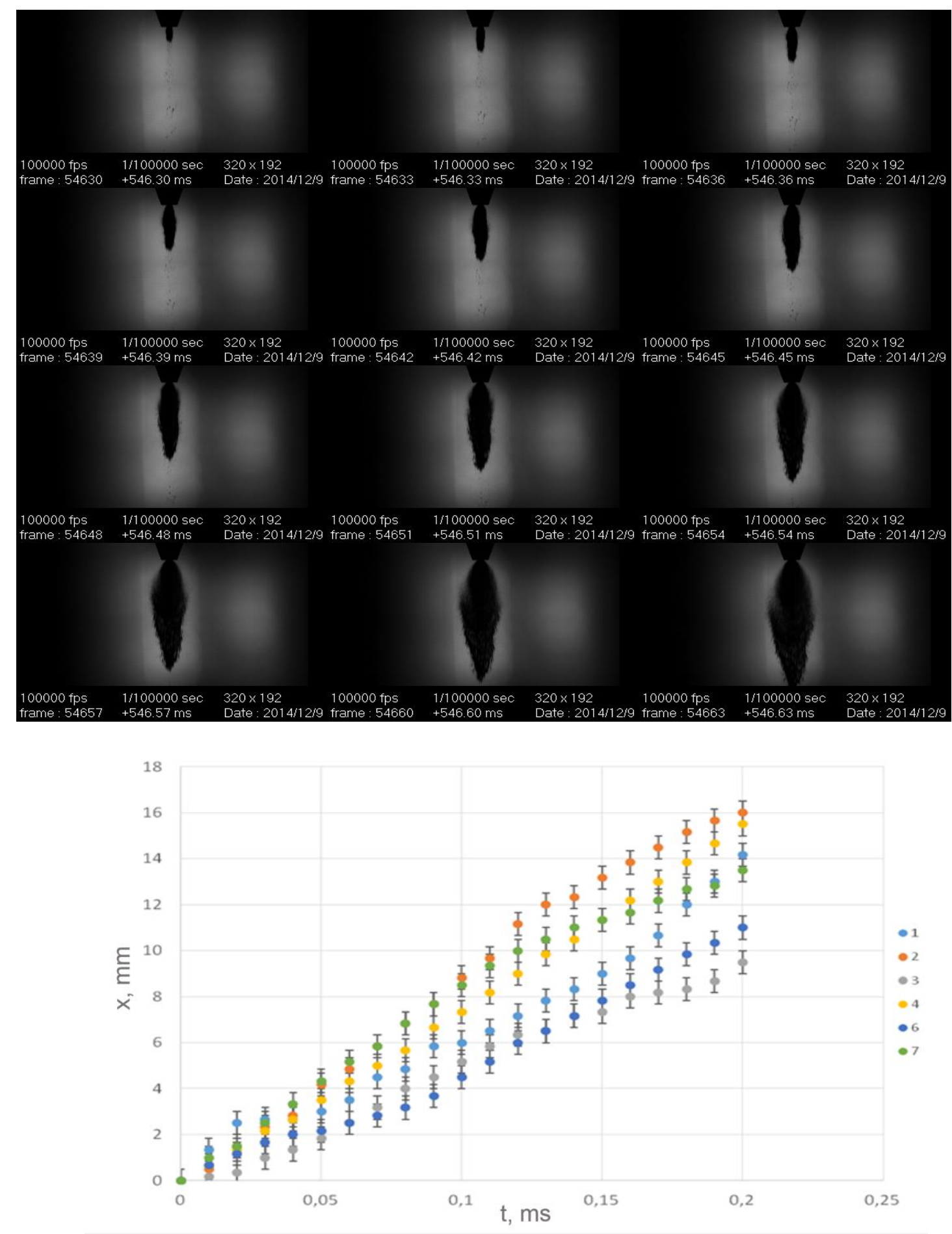

Fig. 4. Visualization of the leader outflow and jet formation process (top) and time dependence of the leader position (bottom). 


\subsection{1/qirt.2016.158}

\section{Experimental results}

In order to study the jet formation process, a high-speed camera with framing rate 100.000 frames per second, resolution $640 \times 376$ pixels and exposure time $1 \mu \mathrm{s}$ was used. The jet configuration in the top area varied under different experimental conditions. A set of images is presented in Fig. 4 (time between two frames is 0.03 ms). The vertical position of a jet leader was determined by a boundary point of a non-transparent area in vertical line - flow axis of symmetry; the measurement accuracy was $1 \mathrm{~mm}$. Video data and images were processed, high-speed water jet outflow spatial-temporal characteristics were determined as well as the stationary mode establishing process. The leader initiation, the jet formation process, and the dynamic characteristics of its travel with acceleration were quantitatively analyzed. It was found that jet leader speed changes from 30 to $270 \mathrm{~m} / \mathrm{s}$ [6].

The thermographic analysis of the waterjet is performed using a calibrated infrared camera FLIR Systems SC7700. Its recording rate can be increased up to $400 \mathrm{~Hz}$ by decreasing the frame size. High-speed thermography method allowed visualizing the dynamics of the radiation from the high-speed water jets in air. Since water is opaque in the mid-wave infrared spectral band, the thermal radiation is captured from the jet periphery and its air-water interface. The heat transfer with the ambient air can be neglected due to negligible temperature differences and the use of insulation.

We investigated the following quantitative characteristics: dynamics of the radiation (temperature pulsations depending on time); spatial distribution of radiation in the jet cross-sections depending on the distance from the nozzle; temperature distribution along the jet axis.
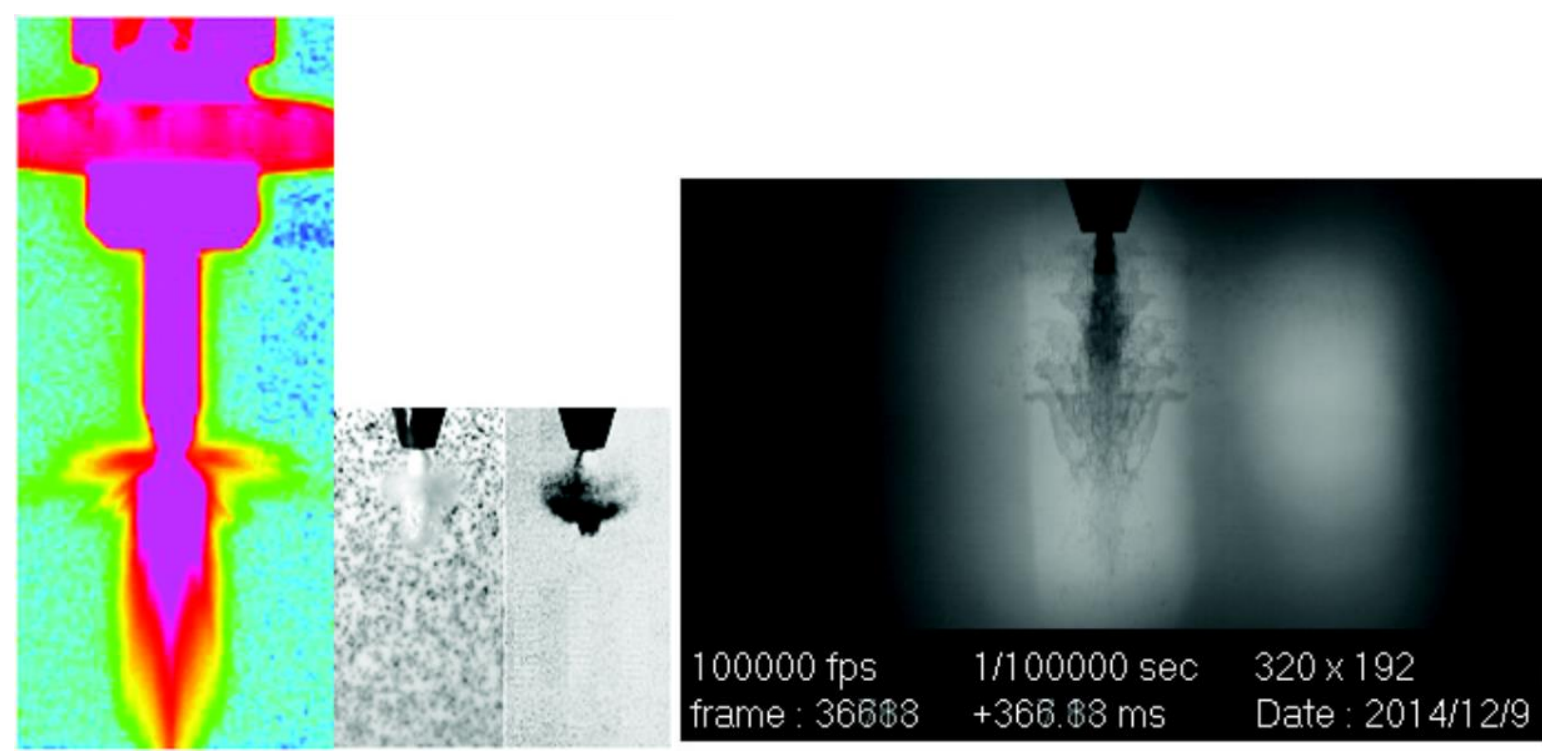

Fig. 5. From left to right: thermographic image of the waterjet head and instant images from the FASTCAM SA5 camera: background oriented; scattered light; refraction and absorption superimposed images.

The camera Photron FASTCAM SA5 provides a temporal resolution up to $1000000 \mathrm{fps}$ with an exposure of 1 $\mu \mathrm{s}$, whereas the FLIR SC7700 thermal imaging camera provides a resolution up to $400 \mathrm{fps}$ with an exposure of $1 \mathrm{~ms}$. Thus, the integrated thermal image of a jet passing in $1 \mathrm{~ms}$ contains a number of instant images equal to 1 thermographic image (Fig. 5). So, the thermographic temperature measurements are integrated during the jet movement in time recorded by 100 shadow images, and we can follow the jet configuration deformation during this period.

Fig. 6 (left) shows an example of typical thermal images of the waterjet starting process (non-stationary mode) and thermal signal curves obtained for 3 points using the Altair processing software. The analysis of the thermograms showed that, when exiting the nozzle, the jet has a single temperature oscillation with an amplitude of about $2-3{ }^{\circ} \mathrm{C}$ during the first $0.1 \mathrm{~s}$ from the start (Fig. 6) Then the jet reaches a stationary state. 

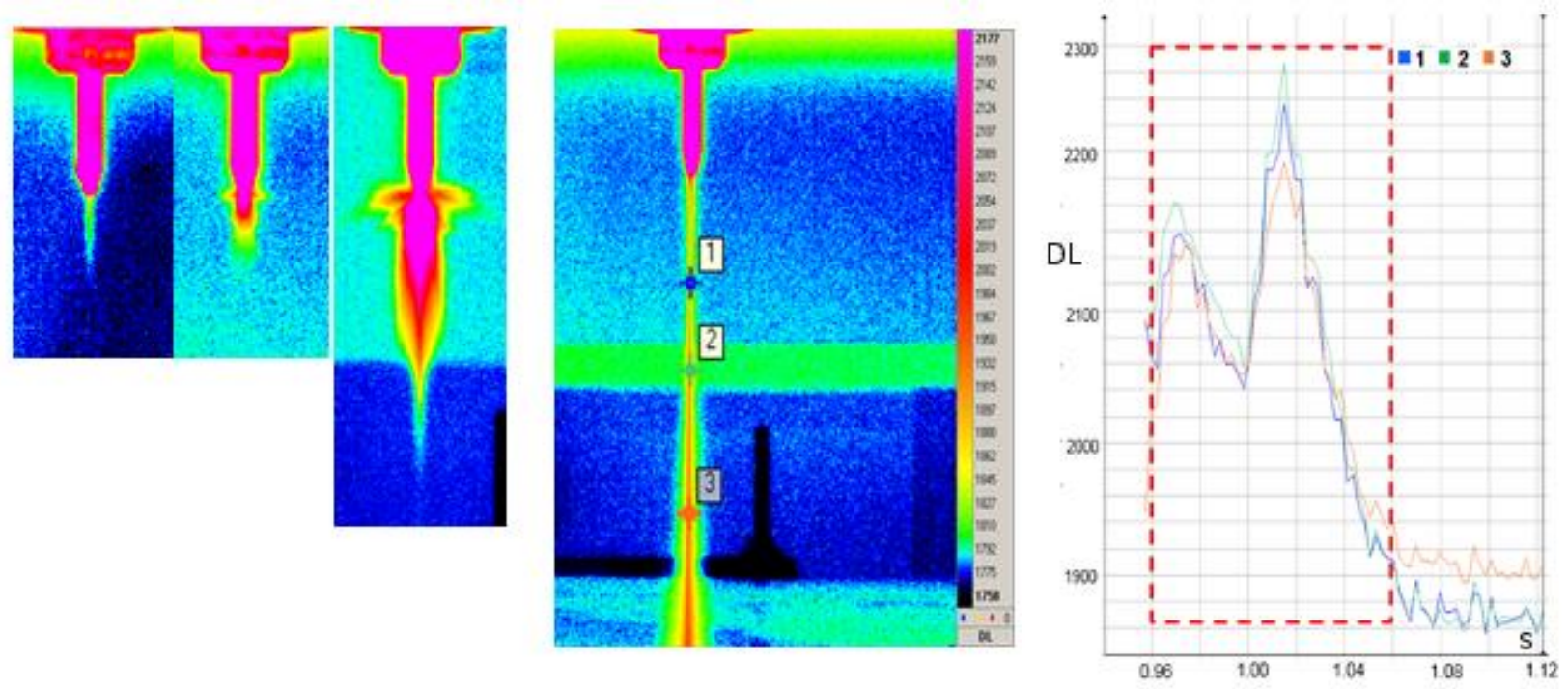

Fig. 6. Thermographic images of the waterjet (left) and thermal radiation evolution versus time at 3 selected points $0.16 \mathrm{~s}$ from the jet start-up (right).

The temperature distribution across the jet in a stationary operating regime was analyzed. Fig. 7 shows characteristic curves of the radiation units (digital level, DL) at different distances from the nozzle exit. The thermal radiation was recorded from the outer interface of the high-speed jet. The recording duration was 5 seconds, and the results were averaged among the frames. The ambient temperature was $10^{\circ} \mathrm{C}$. The observed temperature changes along the central axis of the jet were $15-23^{\circ} \mathrm{C}$. The presence of the minimum in the temperature distribution along the jet axis is due to the nature of its spatial structure - the ratio of the droplet layer around the jet core. The radiation reflection from bump area also affects the thermal measurements.
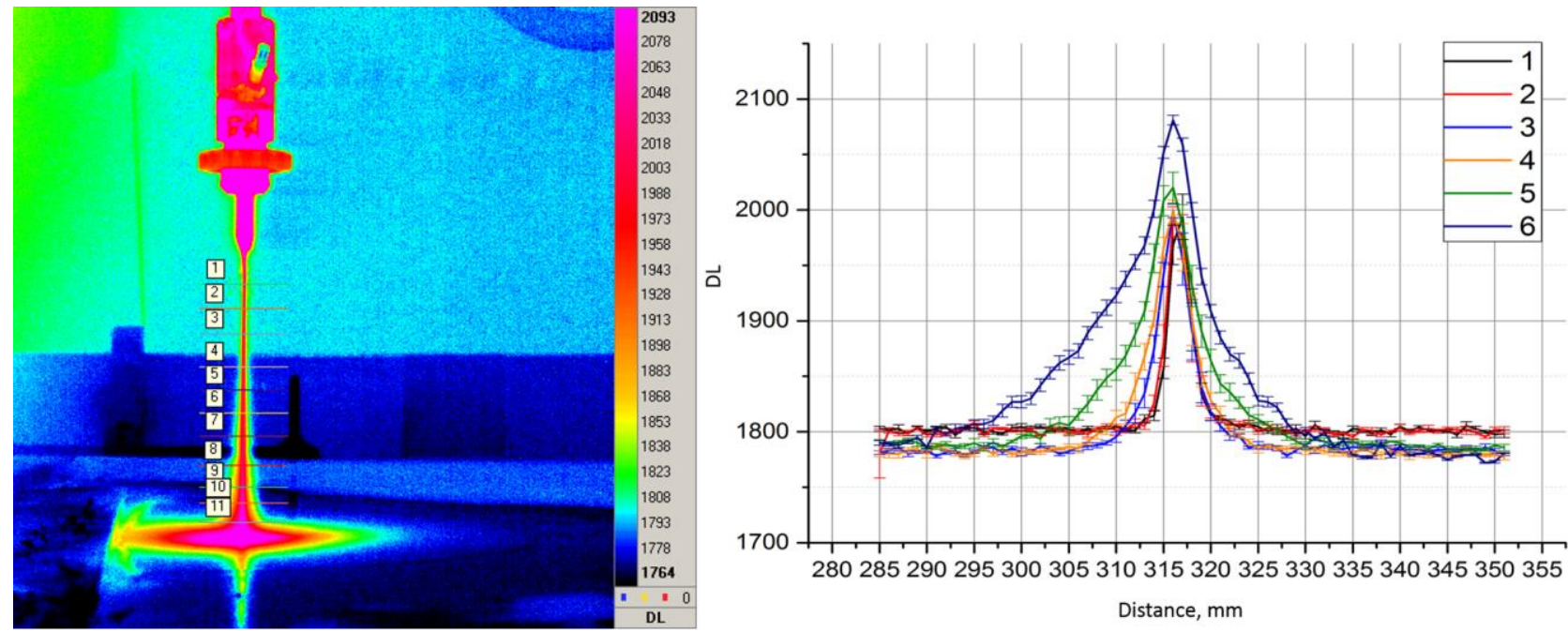

Fig. 7. Distribution of the averaged thermal signal across the jet at different distances from the nozzle exit.

\section{Conclusion}

By monitoring the thermal radiation emitted from a water cutting jet and a material under an impact load, it is possible to gain information on the impacting process. Videos of an AWJ exiting the nozzle recorded by high-speed camera were compared to videos from a thermal imaging camera with time resolution of up to $400 \mathrm{~Hz}$. The nonstationary jet starting process was analyzed. A single temperature oscillation with a $2-3^{\circ} \mathrm{C}$ amplitude during the first $0.1 \mathrm{~s}$ after the jet start-up was detected. The temperature distribution across the jet depending on the distance from the nozzle exit in a stationary mode was also measured and analyzed. 
Acknowledgment. The authors acknowledge support from M.V. Lomonosov Moscow State University Program of Development and RFBR grant 16-38-60186.

\section{REFERENCES}

[1] Summers D.A., "Waterjetting Technology". E \& FN Spon, London, 1995.

[2] Zelenak M., Foldyna J., Scucka J., Hloch S., Riha Z., "Visualisation and measurement of high-speed pulsating continuous water jets". Measurement, vol. 72, pp. 1-8, 2015.

[3] Matthujak A., Pianthong K., Takayama K., Milton B. E., "Experimental Study of Ignition over Impact-Driven Supersonic Liquid Fuel Jet”, 2013.

[4] Kovacevic R., Mohan R., Beardsley H.E., "Monitoring of Thermal Energy Distribution in Abrasive Waterjet Cutting Using Infrared Thermography”. ASME Journal of Manufacturing Science and Engineering, 118 (4), pp. 555-563, 1996.

[5] Lebara A., Junkara M., Poredošb A., Cvjeticanina M., "Method for online quality monitoring of AWJ cutting by infrared thermography”. CIRP Journal of Manufacturing Science and Technology, vol. 2, №3, pp. 170-175, 2010.

[6] Znamenskaya I.A., Naumov D.S., Nersesyan D.A., Sysoev N.N., Shirshov Y.N., "Waterjet cutting machines high speed water jets dynamic characteristics research". The 13th Asian Symposium on Visualization, Novosibirsk, Russia, 22-26 June 2015. 